PROCEEDINGS OF THE

AMERICAN MATHEMATICAL SOCIETY

Volume 128, Number 9, Pages 2753-2761

S 0002-9939(00)05279-5

Article electronically published on March 1, 2000

\title{
INDEPENDENT RANDOM CASCADES ON GALTON-WATSON TREES
}

\author{
GREGORY A. BURD AND EDWARD C. WAYMIRE
}

(Communicated by Stanley Sawyer)

\begin{abstract}
Consider an independent random cascade acting on the positive Borel measures defined on the boundary of a Galton-Watson tree. Assuming an offspring distribution with finite moments of all orders, J. Peyrière computed the fine scale structure of an independent random cascade on Galton-Watson trees. In this paper we use developments in the cascade theory to relax and clarify the moment assumptions on the offspring distribution. Moreover a larger class of initial measures is covered and, as a result, it is shown that it is the Hölder exponent of the initial measure which is the critical parameter in the Peyrière theory.
\end{abstract}

We start by defining the space of Galton-Watson trees and introducing some related terminology.

\section{Galton-Watson trees}

Let $\mathbb{T}$ be the space of labelled tree graphs rooted at $\phi$. An element $\tau$ of $\mathbb{T}$ is a set of finite sequences of positive integers $\left\langle v_{1}, v_{2}, \ldots, v_{n}\right\rangle \in \tau$ such that:

(i) $\phi \in \tau$ is coded as the empty sequence.

(ii) If $\left\langle v_{1}, \ldots, v_{k}\right\rangle \in \tau$, then $\left\langle v_{1}, \ldots, v_{i}\right\rangle \in \tau \forall 1 \leq i \leq k$.

(iii) If $\left\langle v_{1}, v_{2}, \ldots, v_{n}\right\rangle \in \tau$, then $\left\langle v_{1}, \ldots, v_{n-1}, j\right\rangle \in \tau \forall 1 \leq j \leq v_{n}$.

If $\left\langle v_{1}, \ldots, v_{n}\right\rangle \in \tau$, then $\left\langle v_{1}, \ldots, v_{n-1}\right\rangle \in \tau$ is referred to as the parent vertex to $\left\langle v_{1}, \ldots, v_{n}\right\rangle$. Edges are assigned to vertices by requiring that a pair of vertices is connected by an edge if and only if one of them is parent to the other. In this way edges may also be identified with the (unique) non-parental or descendant vertex. This specifies the graph structure of $\tau$ and makes $\tau$ a rooted connected graph without cycles. $\mathbb{T}$ may be viewed as a complete metric space with metric $\rho\left(\tau_{1}, \tau_{2}\right):=\frac{1}{1+\sup \left\{n: \tau_{1}\left|n=\tau_{2}\right| n\right\}}$, where $\tau \mid 0=\emptyset$, and $\tau \mid n:=\left\{\left\langle v_{1}, \ldots, v_{k}\right\rangle \in \tau: k \leq\right.$ $n\}, n \geq 1$. The countable dense subset $\mathbb{T}_{0}$ of finite labelled tree graphs rooted at $\emptyset$ makes $\mathbb{T}$ a Polish space. An important class of probability distributions on the Borel sigma field $\mathcal{B}(\mathbb{T})$ of $\mathbb{T}$ for this paper is the Galton-Watson distribution with single progenitor and offspring distribution $p_{k}, k=0,1, \ldots$, for which the probability

Received by the editors May 14, 1998 and, in revised form, October 8, 1998.

2000 Mathematics Subject Classification. Primary 60G57, 60G30, 60G42; Secondary 60K35.

(C)2000 American Mathematical Society 
assigned to a ball $B\left(\tau, \frac{1}{N}\right), \tau \in \mathbb{T}_{0}, N \in\{1,2, \ldots\}$, is

$$
G W\left(B\left(\tau, \frac{1}{N}\right)\right):=\prod_{v \in \tau \mid(N-1)} p_{l(v)},
$$

where $l(v)$ is the total number of offspring at $v$, i.e. $l(v)=\#\{j:\langle v, j\rangle \in \tau\}$.

In what follows we will consider the Galton-Watson distributed trees with generic offspring number $L$ distributed according to $P(L=k)=p_{k}, k=0,1 \ldots, \sum_{0}^{\infty} p_{k}=$ $1, p_{0}=0$, and having finite mean $m$. The condition $p_{0}=0$ assures non-degeneracy of $\tau$ and most importantly guarantees an infinite line of descent for every vertex of the tree. We generally reserve the letters $\xi, \eta$ to denote infinite rays descending from the root of $\tau$, whereas vertices of $\tau$ are generally denoted by $u, v$. However, although finite rays descending from the root are just as easily represented by vertices, we sometimes use the ray notation as a matter of convenience in describing finite rays. This is particularly useful when a formula depends on the possible finite truncations of a given infinite ray. Write $u \rightarrow v$ if $u$ is a parent to $v$. Let $|u|$ denote the distance from the root to $u$ (height), and let $\xi \wedge \eta$ denote the farthest vertex from the root common to $\xi, \eta$. Write $\eta \mid n$ to reference the vertex $\left\langle\eta_{1}, \eta_{2}, \ldots, \eta_{n}\right\rangle$ in the $n$th generation and $\eta \mid 0:=\emptyset$. Also write $v \in \xi$ to mean $v=\xi \mid n$ for some $n$. For $\eta \mid n=\left\langle\eta_{1}, \eta_{2}, \ldots, \eta_{n}\right\rangle$ let $\eta \mid n * j=\left\langle\eta_{1}, \eta_{2}, \ldots, \eta_{n}, j\right\rangle$.

The boundary of a tree $\tau$, denoted by $\partial \tau$, is the set of all infinite rays descending from the root of $\tau$. The boundary is a subset of the metric space $T=\mathbb{N}^{\mathbb{N}}$ with metric $d(\eta, \xi)=e^{-\sup \{n: \eta|n=\xi| n\}}$. The ball of radius $r$ centered at $\xi$ in this metric space will be denoted $B_{r}(\xi)$. Note the two spaces $T$ and $\mathbb{T}$ are quite distinct. A non-negative function $\theta$ defined on the vertices of $\tau$ is a flow if $\theta$ satisfies

$$
\theta(u)=\sum_{v: u \rightarrow v} \theta(v), \forall u \in \tau .
$$

$\theta(v)=\Lambda(\xi \in \partial \tau: v \in \xi)$ gives a one-to-one correspondence between the positive Borel measures $\Lambda$ on $\partial \tau$ and the flows on $\tau$.

The Hölder exponent at $\xi$ of a probability measure $\Lambda$ defined on $\partial \tau$ is defined by

$$
H \ddot{o}(\Lambda)(\xi):=\lim _{r \downarrow 0} \ln \left(\frac{1}{\Lambda\left(B_{r}(\xi)\right)}\right) / \ln \left(\frac{1}{r}\right),
$$

whenever the limit exists. If $\theta(v)$ is the flow corresponding to $\Lambda$, then this definition reduces to

$$
H \ddot{o}(\Lambda)(\xi):=\lim _{n \rightarrow \infty} \frac{1}{n} \ln \frac{1}{\theta(\xi \mid n)}, \xi \in \partial \tau
$$

Finally, define the carrying dimension of probability measure $\mu$ on an arbitrary metric space $(T, \mathcal{B})$ to be $\operatorname{dim}(\mu):=\inf \left\{\beta: \exists B \in \mathcal{B}, \operatorname{dim}_{H}(B)=\beta, \mu(B)=1\right\}$, where $\operatorname{dim}_{H}(B)$ is the Hausdorff dimension of $B \in \mathcal{B}$.

We close this section with a few examples of positive Borel measures on the boundary of Galton-Watson trees which are central to this paper.

Example 1.1. Given a Galton-Watson tree $\tau$, with the offspring distribution having a finite first moment, there is a natural measure $\Lambda(d \xi, \tau)$ on the boundary of $\tau$ defined by

$$
\Lambda(\xi \in \partial \tau: v \in \xi)=\frac{1}{\prod_{k=0}^{|v|-1} l\left(\left\langle v_{0}, \ldots, v_{k}\right\rangle\right)},
$$


where $\left\langle v_{0}, \ldots, v_{k}\right\rangle$ is the empty sequence $\emptyset$ in the case $k=0$. Since under the Galton-Watson measure, the random variables $\log l(\eta \mid k)$ are iid $\forall \eta \in \partial \tau$, the strong law of large numbers gives

$$
H \ddot{o}(\Lambda)(\eta)=\lim _{n} \frac{1}{n} \ln \prod_{k=0}^{n-1} l(\eta \mid k)=\lim _{n} \frac{1}{n} \sum_{k=0}^{n-1} \ln l(\eta \mid k)=\mathbb{E} \ln (L),
$$

GW-a.s. and independent of $\eta \in \partial \tau$. This measure is the cascade initiator, in the sense defined below in Section 2, which was considered in 6].

Several more examples of positive Borel measures on the boundary of GaltonWatson trees for which the Hölder exponent exists can be found in [5]. We will mention only one of them here.

Example 1.2 (Limit Uniform Measure). Let $\tau$ be a Galton-Watson tree. Here and throughout let $Z_{n}$ be the size of its $n$th generation. Assume $\mathbb{E}\left(Z_{1} \log Z_{1}\right)$ is finite. Also, for an arbitrary vertex $v$ in $\tau$ let $Z_{n}(v)$ be the number of vertices in the $n$th generation of $\tau$ which descended from $v,|v|<n$. Then

$$
U N I F_{\tau}(v)=\lim _{n \rightarrow \infty} \frac{Z_{n}(v)}{Z_{n}}
$$

is a flow on $\tau$. A significant result of [5] was to relax the moment condition from $\mathbb{E} Z_{1}\left(\ln Z_{1}\right)^{2}<\infty$ of [2] to $\mathbb{E}\left(Z_{1} \ln Z_{1}\right)<\infty$ and show that the corresponding random measure $U(d \xi, \tau)$ on $\partial \tau$, called limit uniform measure, has Hölder exponent $\ln (m) \mathrm{GW}$-a.s.; in particular a constant function of $\eta$, as was the case in Example 1.1. The corresponding result for $\mathbb{E} Z_{1}<\infty$ but $\mathbb{E}\left(Z_{1} \ln Z_{1}\right)=\infty$ appears to be unsettled.

\section{INDEPENDENT CASCADES}

A theory of positive T-martingales was introduced in [3] as the general framework for independent multiplicative cascades and random coverings. For basic definitions, let $T$ be an arbitrary locally compact metric space with Borel sigma field $\mathcal{B}$, and let $(\widetilde{\Omega}, \widetilde{\mathcal{F}}, \widetilde{P})$ be a probability space together with an increasing sequence $\widetilde{\mathcal{F}}_{n}, n=$ $1,2, \ldots$, of sub-sigma fields of $\widetilde{\mathcal{F}}$. A positive $T$-martingale is a sequence $\left\{Q_{n}\right\}$ of non-negative $\mathcal{B} \times \widetilde{\mathcal{F}}$-measurable functions on $T \times \widetilde{\Omega}$ such that

(i) For each $t \in T,\left\{Q_{n}(t, \cdot): n=1,2, \ldots\right\}$ is a martingale adapted to $\widetilde{\mathcal{F}}_{n}, n=$ $1,2, \ldots$;

(ii) For $\widetilde{P}$-a.s. $\widetilde{\omega} \in \widetilde{\Omega},\left\{Q_{n}(\cdot, \widetilde{\omega}): n=1,2, \ldots\right\}$ is a sequence of Borel measurable non-negative real-valued functions on $T$.

Let $M^{+}(T)$ denote the space of positive finite Borel measures on $T$ and suppose that $\left\{Q_{n}(t)\right\}$ is a positive $T$-martingale. For $\sigma \in M^{+}(T)$, let $\sigma_{n}:=Q_{n} \sigma$ denote the random measure defined by $Q_{n} \sigma<<\sigma$ and $\frac{d Q_{n} \sigma}{d \sigma}(t):=Q_{n}(t), t \in T$. Denote the space of bounded continuous functions on $T$ by $C_{B}(T)$. Then, since $\int_{T} f Q_{n} d \sigma$ is an $L^{1}$-bounded martingale for each bounded measurable function $f$, one obtains, essentially by the martingale convergence theorem, a random Borel measure $Q_{\infty} \sigma$ such that with probability one (see [3] for details),

$$
\lim _{n \rightarrow \infty} \int_{T} f(t) Q_{n} \sigma(d t)=\int_{T} f(t) Q_{\infty} \sigma(d t), \quad f \in C_{B}(T) .
$$


The measure $\sigma$ is referred to as the cascade initiator. As suggested by the notation, one may view $\sigma \rightarrow \sigma_{\infty} \equiv Q_{\infty} \sigma$ as a random operator acting on $M^{+}(T)$. This notation will be used throughout this paper.

Moreover this extends to include random initiator measures $\sigma=\Lambda$ on $T$. Random initiator measures on $T$ are jointly measurably defined on $T \times \mathbb{T}$, conditionally independent of $\left\{Q_{n}\right\}$ given $\left\{\widetilde{\mathcal{F}}_{n}\right\}$. This includes Examples 1.1, 1.2 above. We will say that such random measures are adapted. Examples 1.1, 1.2 will provide examples of adapted random measures in the specific framework of interest in this paper. Namely, let $\left\{W_{v}: v \in \mathbb{N}^{k}, k \geq 0\right\}$ be iid non-negative random variables with mean one defined on a probability space $(\Omega, \mathcal{F}, P)$, which will serve as the cascade generators. Define a probability space $(\widetilde{\Omega}=\Omega \times \mathbb{T}, \widetilde{\mathcal{F}}=\mathcal{F} \times \mathcal{B}(\mathbb{T}), \widetilde{P}=P \times G W)$, and a filtration $\widetilde{\mathcal{F}}_{n}=\mathcal{F}_{n} \times \mathcal{B}(\mathbb{T})$, where $\mathcal{F}_{n}=\sigma\left\{W_{v}:|v| \leq n\right\}$. A Random Cascade on the boundary of a Galton-Watson tree is a random measure on the metric space $T=\mathbb{N}^{\mathbb{N}}$ carried by a random subset $\partial \tau \subseteq T$ and defined on the probability space $(\widetilde{\Omega}, \widetilde{\mathcal{F}}, \widetilde{P})$ such that $\tau$ has distribution $G W(\cdot)$ and the cascade generators $\left\{W_{v}: v \in \mathbb{N}^{k}, k \geq 0\right\}$ are independent of $\tau$ with distribution $P$. In particular, letting $\Lambda(d \xi, \tau)$ be an adapted positive Borel measure concentrated on $\partial \tau$, the Random Cascade on $\partial \tau$ is the positive $T\left(=\mathbb{N}^{\mathbb{N}}\right)$-martingale with respect to $\widetilde{\mathcal{F}}_{n}:=\mathcal{F}_{n} \times \mathcal{B}(\mathbb{T})$, where $\mathcal{F}_{n}=\sigma\left\{W_{v}:|v| \leq n\right\}$, is defined, suppressing $\widetilde{\omega}=(\omega, \tau) \in \widetilde{\Omega}$, by

$$
Q_{n}(\xi)=\prod_{k=1}^{n} W_{\xi \mid k} 1(\xi \in \partial \tau)
$$

As discussed in the previous section one may now define $\Lambda_{\infty}:=Q_{\infty} \Lambda$ for an adapted initial measure $\Lambda$. It may happen that $\Lambda_{\infty}=0 P$-a.s. and for $G W$-a.e. $\tau \in \mathbb{T}$, which is referred to as degeneracy.

Remark. An equivalent formulation in terms of measures on the unit interval may be given by a tree-induced partition of $[0,1]$ into subintervals; see 6 . However viewing these measures on $\partial \tau$ clarifies the essential structure, though the choice of the metric on $\mathbb{N}^{\mathbb{N}}$ obviously effects dimension calculations, Hölder exponents, etc. Also, viewed in this way and taking logarithms of generators one sees that there is some overlap between multiplicative cascades having strictly positive generators and branching random walks. A basic question in which there is indeed an overlap of interest is one which may be viewed as either survival of the cascade or non-degeneracy of a Laplace transform martingale associated with the branching random walk; see [1].

If in the above setting the offspring distribution is given by $L=b$, a.s., $b \geq 2$, then the resulting cascade is referred to as the Mandelbrot Cascade in [4. In this case the normalized Haar measure on $\partial \tau$ coincides with the measures (1.3) and (1.4). The following theorem of [4] describes the structure of this special case which was considered in [6] for a more general class of random trees. Define

$$
\chi_{b}(h)=\log _{b} \mathbb{E} W^{h}-(h-1), h \geq 0 .
$$

Theorem 2.1 (Kahane-Peyriere). Let $\lambda$ denote normalized Haar measure on $\partial \tau$, where $\tau$ is the (deterministic) b-ary tree, and let $\lambda_{\infty} \equiv Q_{\infty} \lambda$. Then

(i) (non-degeneracy) $\chi_{b}^{\prime}\left(1^{-}\right)<0 \Longleftrightarrow \mathbb{E} \lambda_{\infty}(\partial \tau)>0$.

(ii) (support size) There is a Borel subset $S$ of $\partial \tau$ that a.s. has Hausdorff dimension $D=-\ln (b) \chi_{b}^{\prime}(1)$ for the metric $d(\eta, \xi)$, such that $\lambda_{\infty}(S)=\lambda_{\infty}(\partial \tau)$, and if $B$ is a Borel subset of $\partial \tau$ of Hausdorff dimension less than $D$, then $\lambda_{\infty}(B)=0$. 
Remark. The original version of (iii) carried an extraneous moment condition, namely $E \lambda_{\infty}(T) \ln \lambda_{\infty}(T)<\infty$, which was later removed by the percolation method; [3], 7].

\section{MAIN RESUlts}

Our main goal is to prove the following generalization of Theorem 2.1, and more generally that of $[$, by relaxing the moment conditions on the offspring distribution to finite mean. To this end, in this section the cascade initiator is the random measure $\Lambda$ defined in Example 1.1.

Theorem 3.1. Let $\tau \in \mathbb{T}$ be a Galton-Watson tree with offspring $L$ having a finite first moment. Define

$$
\bar{\chi}(h)=\ln \mathbb{E} W^{h}-H \ddot{o}(\Lambda)[h-1], h \geq 0,
$$

where $H \ddot{o}(\Lambda)=\ln (\mathbb{E} L)$. Then

(i) (non-degeneracy) $\bar{\chi}_{b}^{\prime}\left(1^{-}\right)<0 \Longleftrightarrow \mathbb{E} \Lambda_{\infty}(\partial \tau)>0$.

(ii) (support size) For $G W$-a.e. $\tau$, there is a Borel subset $S$ of $\partial \tau$ that has Hausdorff dimension $D=-\bar{\chi}_{b}^{\prime}(1)$ a.s. such that $\Lambda_{\infty}(S)=\Lambda_{\infty}(\partial \tau)$, and if $B$ is a Borel subset of $\partial \tau$ of Hausdorff dimension less than $D$, then $\Lambda_{\infty}(B)=0$.

Remark. This generalizes the theorem of Peyrière [6] in which he extended the theory in [4] to Galton-Watson trees whose offspring distribution has a finite probability generating function in a neighborhood of one. Note that the result essentially extends the theory for $b$-ary cascades by replacing the branching number $b$ by $\ln (\mathbb{E} L)$, as long as $\mathbb{E} L<\infty$. In Theorem 4.1 of the next section we will extract the essential aspects of our result to also allow for a larger class of initial measures. Also, in view of the generality of the theory in [8], one may obtain corresponding results for various dependent cascades by this same approach.

To prove Theorem 3.1 we use the techniques developed in [7, 8]. The basic idea to compute non-degeneracy criterion is to construct a size biased change of measure $\mathcal{Q}_{\infty}$ on $\Omega \times T \times \mathbb{T}$, calculate bounds for the total cascade mass under $\mathcal{Q}_{\infty}$, and then relate these to the expected total mass under $P$ via a Radon-Nikodym derivative. The main idea for computing fine scale structure is the percolation method. In particular the percolation method reduces the problem of calculating Hausdorff dimension of a non-degenerate cascade to that of finding a critical probability below which the cascade generators may be independently set to zero and the percolated cascade remain non-degenerate, i.e. solved by non-degeneracy criterion.

For this problem the "right" size biased measure is defined by the following disintegration formula on $\Omega \times T \times \mathbb{T}$,

$$
\mathcal{Q}_{\infty}(d \omega \times d \xi \times d \tau)=P_{\xi}(d \omega) \Lambda(d \xi, \tau) G W(d \tau),
$$

where $P_{\xi}$ is the distribution of the cascade generators $\left\{W_{v}\right\}$ size-biased along $\xi$ as defined by (3.2). That is, under $P_{\xi},\left\{W_{v}\right\}$ are independent and, letting $q(d x)$ denote the distribution of $W_{v}$ 's under $P, W_{v}$ 's are distributed independently as $q(d x)$ if $v \notin \xi$, and as $x q(d x)$ if $v \in \xi$. Specifically $P_{\xi}<<P$ on $\mathcal{F}_{n}$ with

$$
\left.\frac{d P_{\xi}}{d P}\right|_{\mathcal{F}_{n}}=\prod_{j \leq n} W_{\xi \mid j}
$$

Note that the cascade generators $W_{v}$ along $\xi$ are strictly positive with size-biased probability one; i.e. $P_{\xi}\left(W_{v}=0\right)=0$ for $v \in \xi$. 
The next theorem provides the link between $P$ and the size-biased change of measure $\mathcal{Q}_{\infty}$.

Theorem 3.2 (A Lebesgue Decomposition). Let $\pi_{\widetilde{\Omega}}$ denote the coordinate projection map of $\Omega \times T \times \mathbb{T}$ onto $\widetilde{\Omega}=\Omega \times \mathbb{T}$. Then,

$$
d \mathcal{Q}_{\infty} \circ \pi_{\widetilde{\Omega}}^{-1}=\Lambda_{\infty}(T) \mathbf{1}\left(\Lambda_{\infty}(T)<\infty\right) d(P \times G W)+\mathbf{1}\left(\Lambda_{\infty}(T)=\infty\right) d \mathcal{Q}_{\infty} \circ \pi_{\widetilde{\Omega}}^{-1},
$$

where $\Lambda_{\infty}=Q_{\infty} \Lambda$.

Remark. The proof of Theorem 3.2 is identical to the analogous results of Theorem 2.2 and Corollary 2.1 proved in [8], and hence will be omitted.

Theorem 3.3 (A First Departure Submartingale Bound). Fix $\xi \in T=\mathbb{N}^{\mathbb{N}}$. Let $\widetilde{\mathcal{F}}_{\xi, n}:=\sigma\left\{W_{\xi \mid i}, W_{v}: i \geq 0,|v| \leq n\right\} \times \mathcal{B}$. Fix a sequence $c_{k} \geq 0$, such that $\sum_{k} c_{k}<$ $\infty$. Let $\theta_{\Lambda}$ be the flow associated with $\Lambda$. Then, sample-pointwise on $\widetilde{\Omega}$, one has

$$
\begin{aligned}
\theta_{\Lambda}(\xi \mid n) \prod_{i \leq n} W_{\xi \mid i} 1(\xi \in \partial \tau) \leq \Lambda_{n}(\partial \tau) 1(\xi \in \partial \tau) \leq & \theta_{\Lambda}(\xi \mid n) \prod_{i \leq n} W_{\xi \mid i} 1(\xi \in \partial \tau) \\
& +\sup _{j}\left(\frac{\theta_{\Lambda}(\xi \mid j) \prod_{i \leq j} W_{\xi \mid i}}{c_{j}}\right) M_{n},
\end{aligned}
$$

where, for $\widetilde{\omega}=(\omega, \tau)$,

$$
M_{n}(\widetilde{w})=\sum_{j=1}^{n-1} c_{j} \sum_{\substack{|\eta|=n \\|\eta \wedge \xi|=j}} \prod_{i=j}^{n-1} \frac{1}{l(\eta \mid i, \tau)} \prod_{i=j+1}^{n} W_{\eta \mid i}(\omega) 1(\xi \in \partial \tau)
$$

is a non-negative $P_{\xi} \times G W$-submartingale with respect to $\widetilde{\mathcal{F}}_{\xi, n}$. Furthermore, $\lim _{n} M_{n}$ exists $P_{\xi} \times G W$-a.s.

Proof. The proof is similar to the one given in 8 for the deterministic $b$-ary tree; however one needs to be a little bit more careful since in general $\Lambda$ is not a Haar measure on $\partial \tau$.

We have, writing $\partial \tau=\bigcup_{|v|=n}\{\xi \in \partial \tau: v \in \xi\}$, for $(\omega, \tau)$ such that $\xi \in \partial \tau$,

$$
\Lambda_{n}(\partial \tau)=\sum_{|v|=n} \theta_{\Lambda}(v) \prod_{k=1}^{n} W_{v \mid k}
$$

where, in the notation of Section $2, \Lambda_{n}=Q_{n} \Lambda$. Therefore, the lower bound follows right away from positivity. To get the upper bound, for sample points $(\omega, \tau)$ such that $\xi \in \partial \tau$, write

$$
\begin{aligned}
& \Lambda_{n}(\partial \tau)=\sum_{|\eta|=n} \theta_{\Lambda}(\eta \mid n) \prod_{k=1}^{n} W_{\eta \mid k}=\theta_{\Lambda}(\xi \mid n) \prod_{k=1}^{n} W_{\xi \mid k}+ \\
& +\sum_{j=1}^{n-1} \sum_{\substack{|\eta|=n \\
|\eta \wedge \xi|=j}} c_{j}^{-1} \theta_{\Lambda}(\xi \mid j) \prod_{i=1}^{j} W_{\xi \mid i} c_{j} \prod_{i=j}^{n-1} \frac{1}{l(\eta \mid i)} \prod_{i=j+1}^{n} W_{\eta \mid i} \\
& \leq \theta_{\Lambda}(\xi \mid n) \prod_{k=1}^{n} W_{\xi \mid k}+\sup _{j}\left(\frac{\theta_{\Lambda}(\xi \mid j) \prod_{k=1}^{j} W_{\xi \mid k}}{c_{j}}\right) M_{n} .
\end{aligned}
$$


Finally, to show that $M_{n}$ is a submartingale, note that $M_{n}$ is $\widetilde{\mathcal{F}}_{\xi, n}$ measurable, $\mathbb{E}_{P_{\xi} \times G W}\left|M_{n}\right|<\infty$ and

$$
\begin{gathered}
\mathbb{E}_{P_{\xi} \times G W}\left(M_{n+1} \mid \widetilde{\mathcal{F}}_{\xi, n}\right)=\mathbb{E}_{P_{\xi} \times G W}\left[\sum_{j=1}^{n} c_{j} \sum_{\substack{|\eta|=n+1 \\
|\eta \wedge \xi|=j}} \prod_{i=j}^{n} \frac{1}{l(\eta \mid i)} \prod_{i=j+1}^{n+1} W_{\eta \mid i} \mid \widetilde{\mathcal{F}}_{\xi, n}\right] \\
=\mathbb{E}_{P_{\xi} \times G W}\left[\sum_{j=1}^{n-1} c_{j} \sum_{\substack{|\eta|=n+1 \\
|\eta \wedge \xi|=j}} \prod_{i=j}^{n} \frac{1}{l(\eta \mid i)} \prod_{i=j+1}^{n+1} W_{\eta \mid i} \mid \widetilde{\mathcal{F}}_{\xi, n}\right]+\left(\frac{c_{n}[l(\xi \mid n)-1]}{l(\xi \mid n)}\right) \\
=M_{n}+\left(\frac{c_{n}[l(\xi \mid n)-1]}{l(\xi \mid n)}\right) .
\end{gathered}
$$

Therefore, $M_{n}$ is a non-negative $P_{\xi} \times G W$-submartingale with the bounded predictable part $\sum_{j=0}^{n-1} \frac{c_{j}[l(\xi \mid j)-1]}{l(\xi \mid j)}$ so that applying Doobs Submartingale Decomposition one sees that $\lim _{n} M_{n}$ exists and is finite $P_{\xi} \times G W$-a.s.

The next result is proven by the same considerations as in Theorem 3.3 but is stated here as an alternative useful way to represent the total mass $\Lambda_{n}(\partial \tau)$ in terms of its associated flow, $\theta$ (see Section 1).

Theorem 3.4. For arbitrary $\xi \in \mathbb{N}^{\mathbb{N}}$ and $n \geq 0$ one has

$$
\Lambda_{n}(\partial \tau) 1(\xi \in \partial \tau)=\sum_{j=0}^{n} \theta_{\Lambda}(\xi \mid j) \prod_{i \leq j} W_{\xi \mid i} M_{n, j}
$$

where

$$
M_{n, j}=\sum_{\substack{|\eta|=n \\|\eta \wedge \xi|=j}} \prod_{i=j}^{n-1} \frac{1}{l(\eta \mid i)} \prod_{i=j+1}^{n} W_{\eta \mid i} 1(\xi \in \partial \tau)
$$

is the total mass in a subtree rooted at $\xi \mid j$.

We are now ready to prove part (i) of Theorem 3.1 .

A sufficient condition for non-degeneracy. Suppose $\mathbb{E}_{P} W \ln W<H \ddot{o}(\Lambda)$. Fix $\epsilon>$ 0 small enough such that $H \ddot{o}(\Lambda)-\mathbb{E}_{P} W \ln W-4 \epsilon>0$, let $c=\exp \{H \ddot{o}(\Lambda)-$ $\left.\left.\mathbb{E}_{P} W \ln W-4 \epsilon\right)\right\}$, and take $c_{j}=c^{-j}$. Fix $\xi \in \partial \tau$. Thus,

$$
\sup _{j} \frac{\theta_{\Lambda}(\xi \mid j) \prod_{k \leq j} W_{\xi \mid k}}{c_{j}}<\infty .
$$

Therefore, using the upper bound in Theorem 3.3, $\Lambda_{\infty}(\partial \tau)<\infty P_{\xi}$-a.s. and thus $P$-a.s. Hence, $\mathbb{E} \Lambda_{\infty}(T)=1$ by Theorem 3.2 .

A necessary condition for non-degeneracy. To see why the entropy condition is also necessary for non-degeneracy, suppose that $\mathbb{E}_{P} W \ln W \geq H \ddot{o}(\Lambda)$. Fix $\xi \in \partial \tau$. If $\mathbb{E}_{P} W \ln W>H \ddot{o}(\Lambda)$, then using the lower bound of Theorem 3.3 one obtains

$$
\begin{aligned}
\Lambda_{n}(\partial \tau) \geq \theta_{\Lambda}(\xi \mid j) \prod_{i \leq j} W_{\xi \mid i} & =\exp \left\{j\left(\frac{1}{j} \sum_{i \leq j} \ln W_{\xi \mid j}-\frac{1}{j} \sum_{i \leq j} \ln l(\xi \mid i)\right)\right\} \\
& =O\left(\exp \left\{-j\left(\mathbb{E}_{P_{\xi}} \ln W_{\xi \mid j}-H \ddot{o}(\Lambda)\right)\right\}\right)
\end{aligned}
$$


as $j$ goes to $\infty, P_{\xi}$-a.s. by the $\operatorname{SLLN}$. Hence $\Lambda_{\infty}(\partial \tau)=\infty P_{\xi^{-a}}$.s., and by Theorem 3.2

$$
\int_{\Omega} \Lambda_{\infty}(\partial \tau) d P(\omega)=0 \Longrightarrow \Lambda_{\infty}(\partial \tau)=0 \quad P \text {-a.s. }
$$

For the critical case, $\mathbb{E}_{P} W \ln W=H \ddot{o}(\Lambda)$, use the Chung-Fuchs criterion applied to the random walk with the increments given by $\ln W_{\xi \mid n}-\ln l(\xi \mid n)$ instead of the SLLN to conclude that $\lim \sup _{n \rightarrow \infty} \Lambda_{n}(\partial \tau)=\infty, P_{\xi}$-a.s.

Carrying dimension. To compute the carrying dimension of the multiplicative cascade we use the percolation method; see [3, [7]. In particular the problem of finding the carrying dimensions for the cascade measure reduces to one of finding the critical surviving parameter $p$ of the percolated cascade $Q_{\infty}^{(p)}\left(Q_{\infty} \Lambda\right)$ with independent generators distributed as the independent product $B W$, with

$$
B= \begin{cases}\frac{1}{p} & \text { with probability } p \\ 0 & \text { with probability } 1-p\end{cases}
$$

In view of the previously computed survival criterion, one has $\mathbb{E}_{P}(B W) \ln (B W)-$ $H \ddot{o}(\Lambda)=0$ if and only if

$$
\operatorname{Dim}=-\ln p=\mathbb{E}_{P} B \ln B=H \ddot{o}(\Lambda)-\mathbb{E}_{P} W \ln W .
$$

\section{The General CASE}

Most of the results in the previous section did not take advantage of the GaltonWatson distribution beyond establishing properties of the underlying measure $\Lambda$. For example, we require that each vertex a.s. be locally finite and have an infinite line of descent, and $\Lambda$ be an adapted random initiator measure with a.s. constant Hölder exponent as defined in Sections 1 and 2. In particular, for a given measure on $\partial \tau$ with the finite Hölder exponent, not depending on $\tau$, one obtains the nondegeneracy criterion and the support size of the cascade measure by first departure considerations. For a given vertex $v \in \tau$, let $\tau_{v}$ be the subtree of $\tau$ rooted at $v$. Then $\theta_{\Lambda}$ restricted to $\tau_{v}$ is a flow on $\tau_{v}$, and hence there exists a corresponding measure $\Lambda_{v}$ on $\partial \tau_{v}$. A closer look at the First Departure Submartingale Bound Theorem reveals that it is a consequence of the following simple fact. For a fixed but arbitrary $\xi \in \tau$,

$$
\theta_{\Lambda}(\xi \mid n)=\theta_{\Lambda}(\xi \mid k) \theta_{\Lambda_{x}}\left(\xi_{k} \mid(n-k)\right), \forall k \in(0, n), n>1,
$$

where $\xi_{k}$ is an infinite ray descending from $\xi \mid k$ such that $\xi_{k}|l=\xi|(k+l), \forall l=$ $0,1, \ldots$

Definition. A measure $\Lambda$ on a boundary of a Galton-Watson tree has a multiplicative flow property iff the corresponding flow $\theta_{\Lambda}$ satisfies (4.1).

A review of the steps in the previous proofs makes it clear that the results hold for an arbitrary distribution of locally finite trees for which one also has an adapted initial measure with a.s. constant Hölder exponent and with the multiplicative flow property. We term such tree distributions and initial measures admissible in the statement of the next theorem. Note that one can do a reduction of the tree by pruning off finite lines of descent and stating results for such reduced trees if necessary. 
Theorem 4.1. Let $\tau \in \mathbb{T}$, and let $\Lambda$ be admissible. Also let

$$
\bar{\chi}(h)=\ln \mathbb{E} W^{h}-H \ddot{o}(\Lambda)[h-1], h \geq 0 .
$$

Then a.s.

(i) (non-degeneracy) upon non-criticality, i.e. $\bar{\chi}^{\prime}\left(1^{-}\right) \neq 0, \bar{\chi}^{\prime}\left(1^{-}\right)<0 \Longleftrightarrow$ $\mathbb{E} \Lambda_{\infty}(\partial \tau)>0$.

(ii) (support size) There is a Borel subset $S$ of $\partial \tau$ a.s. that has Hausdorff dimension $D=-\bar{\chi}_{b}^{\prime}(1)$ such that $\Lambda_{\infty}(S)=\Lambda_{\infty}(\partial \tau)$, and if $B$ is a Borel subset of $\partial \tau$ of Hausdorff dimension less than $D$, then $\Lambda_{\infty}(B)=0$.

Example 4.1. Consider the limit uniform measure $U$ of Example 1.2 on $\partial \tau$. If $\eta \in \partial \tau$ and $n>k>0$, then

$$
\begin{aligned}
U N I F_{\tau}(\eta \mid n) & =\lim _{N} \frac{Z_{N}(\eta \mid n)}{Z_{N}}=\lim _{n} \frac{Z_{n}(\eta \mid k)}{Z_{n}} \lim _{N} \frac{Z_{N}(\eta \mid n)}{Z_{N}(\eta \mid k)} \\
& =U N I F_{\tau}(\eta \mid k) \lim _{N} \frac{Z_{N}(\eta \mid n)}{Z_{N}(\eta \mid k)} .
\end{aligned}
$$

The multiplicative flow property for $U$ now follows since the subtree descending from the vertex $\eta \mid k$ is itself a supercritical Galton-Watson tree. Therefore, Theorem 4.1 holds for the limit uniform measure, with $\bar{\chi}(h)=\ln \mathbb{E} W^{h}-\ln m[h-1]=\ln m \chi_{m}(h)$.

\section{ACKNOWLEDGments}

The authors wish to express their gratitude to an anonymous referee and the associate editor for pointing out some technical errors and generally clarifying the presentation of results in an earlier draft of this paper. This work was partially supported by a grant from the National Science Foundation.

\section{REFERENCES}

[1] Biggins, J., Martingale convergence in the branching random walk, J. Appl. Prob 14 (1977), 25-37. MR 55:6592

[2] Hawkes, J., Trees generated by a simple branching process, J. London Math.Soc. 2(24) (1981), 373-384. MR 83b:60072

[3] Kahane, J.P., Random multiplications, random coverings, and multiplicative chaos, Proc. Special Year in Modern Analysis 137 (1989), 196-255. MR 91e:60152

[4] Kahane, J.P. and J. Peyrière, Sur certaines martingales de B. Mandelbrot, Adv. in Math. 22 (1976), 131-145. MR 55:4355

[5] Lyons, R., R. Permantle and Y. Peres, Ergodic theory on Galton-Watson trees: speed of random walk and dimention of harmonic measure., Ergod. Th. \& Dynam. Sys. 15 (1995), 593-619. MR 96e:60125

[6] Peyriere, J., Calculus de dimensions de Hausdorff, Duke Math J. 44 (1977), 591-601. MR 56:3257

[7] Waymire, E. and S.C. Williams, Multiplicative cascades: Dimension spectra and dependence, The J. of Fourier Analysis and Appl. Special Issue (1995), 589-609. MR 97e:60082

[8] Waymire, E. and S.C. Williams, A cascade decomposition theory with applications to Markov and exchangeable cascades, T.A.M.S. 348(2) (1996), 585-632. MR 96j:60086

Department of Mathematics, University of Washington, Seattle, Washington 98195 E-mail address: burd@math.washington.edu

Current address: Marvell Semiconductor, Inc., 645 Almanor Avenue, Sunnyvale, California 94086

E-mail address: gburd@marvell.com

Department of Mathematics, Oregon State University, Corvallis, Oregon 97331-4605

E-mail address: waymire@math.orst.edu 